\title{
EFFECT OF ATTACHMENT RESILEINCY ON MUSCLE ACTIVITY IN IMPLANT RETAINED MANDIBULAR OVERDENTURE
}

\author{
Nada El Khourzaty*, Nancy N Elsherbini * and Aliaa A Tawfik ${ }^{* *}$
}

\begin{abstract}
Objectives: This study aimed to investigate masticatory muscle activity in patients treated with 2 modalities of implant mandibular overdenture prosthesis.

Methodology: Fourteen completely edentulous male patients were selected and four implants were inserted for each patient. After 3 months of installation, the patients were equally divided into two groups; group I receiving overdentures retained with ball attachments and group II receiving telescopic retained overdentures. Muscle activity was measured at time of denture insertion, two weeks and one month after insertion.
\end{abstract}

Results: The results revealed that muscle activity was significantly lower in telescopic retained overdentures compared to overdentures retained with ball attachments.

Conclusion: Telescopic attachments decreased the effort exerted by the muscles compared to ball and socket attachments.

KEY WORDS: muscular activity, implants, overdenture, telescopic attachments.

\section{INTRODUCTION}

The implant supported overdenture became a reliable mode of treatment for the completely edentulous mandible since they enhance the support, stability as well as retention of the prosthesis and successfully restoring oral function thus permitting better biting and chewing function than conventional complete dentures. ${ }^{1,2}$ It was also concluded that the use of attachments to retain the implant supported prosthesis further improves the biting force and patient's masticatory function and overall satisfaction, regardless of the attachment type used. ${ }^{2-4}$

Stud attachments are one of the most commonly used resilient attachments with implant overdentures due to their ease of handling, cost effectiveness, less technique sensitivity, better stress distribution, and acceptable retention ${ }^{5,6}$. The use of the ball attachments is considered advantageous for optimizing stress and minimizing denture movement, ${ }^{78}$ in addition to a lesser frequency of complications and repairs. ${ }^{9}$

*Lecturer, Removable Prosthodontics, Faculty of Dentistry, Cairo University, Cairo, Egypt

**Lecturer, Clinical Neurophysiology Unit, Faculty of Medicine, Cairo University, Cairo, Egypt 
Telescopic attachments are rigid crowns that have many advantages that may favor its usage in implant supported restorations. They provide excellent frictional retention between the primary and secondary copings in addition to proper force distribution through the relation between the outer crown and the abutment. Also the direction of force applied is vertically applied and so well tolerated and enhances the preservation of bone. ${ }^{10,11}$

Mastication is an important function to maintain, not only for oral health, but also for better life quality. ${ }^{12}$ Chewing efficiency was affected by oralhealth-related quality of life and general health, possibly reflecting the impact of chewing on the type of food, thus, indicating the importance of adequate oral health for general well-being. ${ }^{13}$

EMG (electromyography) measures muscle function and subsequently muscle efficiency which is affected by mandibular movement, masticatory muscle function as well as food texture..$^{14,15}$ Muscles play numerous roles in the stomatognathic system especially during mastication, clenching and chewing. It also contributes effectively in the neuromuscular control of the prosthesis which is a vital influential factor on the retention and stability of removable restorations. 1,16 EMG data are characterized by being repeatable and so has been used to study the masticatory muscle behavior of the stomatognathic system during normal or abnormal conditions as occlusion and bruxism, helping with the diagnosis of orofacial pain, and in evaluation of the efficiency of different treatment modalities in patients with temporomandibular disorders. ${ }^{15}$

Therefore using EMG to evaluate the muscles of mastication is a good dependable way to determine masticatory efficiency. EMG depends on several factors as patients age as well as the psychological factors as fear of pain, fracture, instability of the denture, are considered to have a great influence on muscular activity reduction. ${ }^{17}$

Investigation of the electromyographic (EMG) activity of masseter muscle in patients rehabilitated with implant-supported overdenture during chewing different food consistencies, it was concluded that with implant-retained mandibular overdentures chewing patterns were more regular with higher electrical activity of the masseter muscles, thus providing improved chewing function and comfort. ${ }^{18}$

This study aimed to evaluate the muscular activity using resilient and rigid attachments for implant retained mandibular overdenture.

\section{MATERIALS AND METHODS}

Fourteen male completely edentulous male patients were selected from the outpatient clinic of Prosthodontics Department; Faculty of Oral and Dental Medicine, Cairo University. The patients' age ranged from 55 to 65 years old. Patients were systemically free from any disease that may affect or interfere with dental implant placement and/or osseointegration, in addition to having adequate bone for implant placement. Sufficient inter arch space for overdenture construction with normal maxillomandibular relation. Cooperative patients who followed the instructions and having proper neuromuscular co-ordination were included in the study. Patient history, clinical examination and radiographic assessment were carefully carried out to verify the selection criteria.

A complete denture was fabricated for patients who do not have a denture or had an old unacceptable denture.

The finished lower denture was duplicated and a radio opaque acrylic resin stent was done for each patient. Four small holes were drilled in the stent at the proposed implant sites and a cone beam CT image was obtained.

Four $3.7 \mathrm{~mm}$ diameter implants (Neo Biotech Co. Ltd, Seoul, Korea) were placed with the aid of the surgical guide in the canine and molar region area using sequential drilling after flap reflection. The direction of drilling was kept perpendicular 
to the bone and midway buccolingually, putting in consideration the parallelism between the four implants. Three months following surgical implant installation, limited flap reflection was done for placing the abutments and patients were prepared for the prosthetic phase and randomly divided into two groups seven patients each:

- Group I: Patients of this group received a mandibular overdenture retained by a ball abutment and a nylon matrix(Neo Biotech Co. Ltd, Seoul, Korea). Fig (1“a,b”)

- Group II: Patients of this group received a mandibular telescopic attachment retained overdenture. Fig (1 “c,d”)

Group I procedure: The matrices and housings of the attachment was secured firmly over the abutments, holes corresponding to the housings were opened in the fitting surface of the denture allowing proper seating of the denture without any interference with the housings, as indicated by absence of rocking, pressure indicating paste and proper occlusion. Chemically cured resin was placed in the relieved areas and the denture was seated in the patient's mouth. The resin was left to polymerize while the patient closing in centric jaw relation with gentle pressure. The overdenture was then removed, trimmed and polished with the housings picked up in its fitting surface.

Group II procedure: Impression copings (Neo Biotech, Korea) were screwed to implant abutments, splinted together then impression was made with medium consistency elastomeric material (SPEEDEX, COLTENE ${ }^{\circledR}$, Switzerland) in open tray. The mandibular impression was poured with high strength type III dental stone (ELITE $\mathrm{STONE}^{\circledR}$, Zhermack, Italy). Acrylic trial denture bases with wax rim on were fabricated, then Jaw relation records, face-bow transfer, mounting on a semi-adjustable articulator HANAU $\mathrm{H}$ (Arcon, Louisville, KY USA 40209), setting-up of semi-anatomical cross-linked acrylic teeth in a lingualized occlusion pattern and then Try-in was done. A rubber index was made for the setting up of teeth after the try-in stage. Customized plastics were screwed to the analogues, waxed up with milling wax (PLASTODENT ${ }^{\circledR}$, Degussa. Germany) and milled with milling surveyor(BREDENT BF2, Bredent, Germany) to form parallel walled primary telescopic crowns. Attachment orientation was guided by the rubber index. Investing and casting of wax pattern was done, after then finishing and polishing. Try-in of primary crowns was done in patient's mouth. Then the copings were returned on the cast for the waxing up of the secondary coping and the metal framework. Then casting was made and the framework was verified in the patient's mouth. Finally heat cured acrylic resin was processed on the metallic framework to form the mandibular over-denture.

\section{Measuring the masticatory activity}

Muscular activity, measured in voltage and represented by the root mean square value (RMS), was evaluated by using a digital electromyogram (EMG) (Nihon Kohden, America, INC. USA).

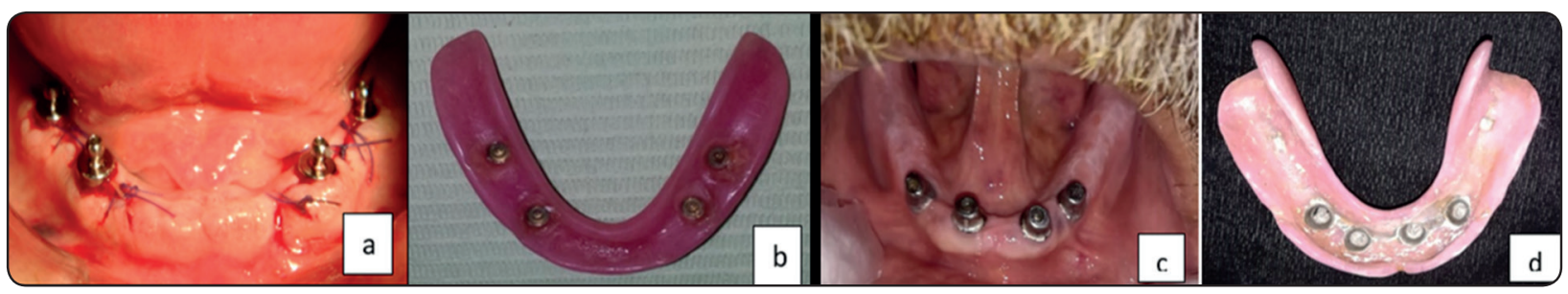

Fig. (1) (a,b,c\&d) 
Measuring was made at the time of denture insertion, two weeks and one month after insertion ${ }^{1}$. The patients were seated in an upright relaxed position during the measuring procedure. Muscle activity was recorded during chewing equal sized tablets (Bredent, Germany) of hard and soft consistencies. Five minutes were left between each record to avoid muscle fatigue. when the EMG rhythm became more stable data were recorded after the first 3 cycles of chewing, when the EMG rhythm became more stable. Five records of the root mean square for each muscle, which represented the total integrated muscle activity, were obtained for each of the above-mentioned performances. After which the mean of the 5 records for each performance was calculated regarding each muscle at the different follow-up periods. A transparent template was prepared to be used at each subsequent follow-up visit to aid in repositioning of surface electrodes in their exact position. Measurements were displayed and saved on a computer. Figure (2)

Data were collected, statistically analyzed using SPSS statistical package (Version 17, Chicago, IL) and represented in tables and figures. Two way ANOVA test was used to detect the difference in the two treatment modalities and the effect on the muscles and Tukey's post hoc test.

\section{RESULTS}

The mean values of recorded EMG muscle activity during soft and hard food activities with ball retained implant overdenture and implant retained telescopic overdenture) along the follow up periods (immediate, two weeks and one month) after insertion.

\section{Effect of attachment type on muscle activity:}

\section{When chewing soft food:}

In case of temporalis muscle: For all follow up periods ( 0,2 weeks and 1 month), the highest mean muscle activity was recorded for the ball retained implant overdenture although the difference was not significant. For the masseter muscle: For all follow up periods $(0,2$ weeks and 1 month), the highest mean muscle activity was recorded for the ball retained implant overdenture, the difference was statistically significant.p $\leq 0.001$

\section{When chewing hard food:}

In case of temporalis muscle: For all follow up periods ( 0,2 weeks and 1 month), the highest mean muscle activity was recorded for the ball retained implant overdenture although the difference was non significant. On the other hand, the masseter

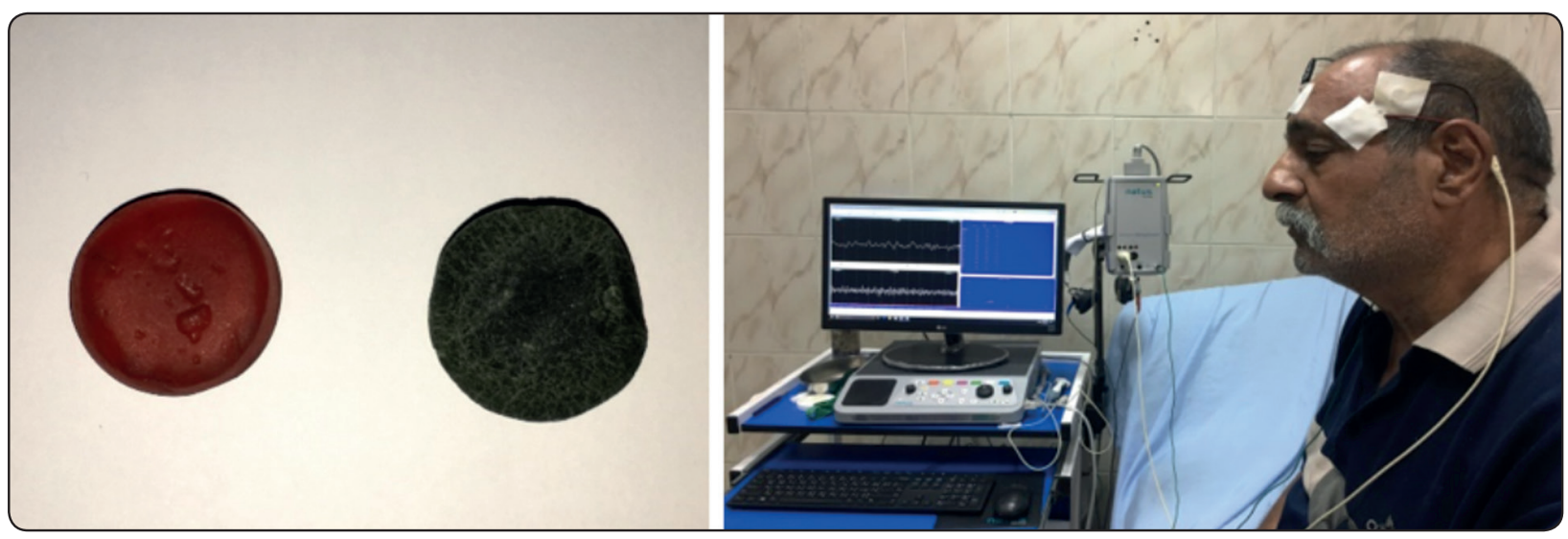

Fig. (2) 
muscle showed through all follow up periods $(0$ ,2 weeks and 1 month), the highest mean muscle activity recorded was for the ball retained implant overdenture, the difference was statistically significant. $\mathrm{p} \leq 0.001$.

\section{Effect of time on muscle activity:}

\section{For telescopic retained implant overdenture with soft food chewing:}

For Temporalis muscle between $(0$ and 2 weeks) follow up periods there was no statistically significant difference in the mean muscle activity. On the other hand there was statistically significant decrease between ( 2 weeks and 1 month) in the mean muscle activity. $\mathrm{p} \leq 0.001$. While for masseter muscle: there was statistically significant decrease in the mean muscle activity through the whole follow up intervals. $p \leq 0.001$

\section{For telescopic retained implant overdenture with hard food chewing:}

For temporalis muscle there was statistically significant decrease in the mean muscle activity between ( 0 and 1 month) intervals. While for masseter muscle there was decrease in the mean muscle activity through the whole follow up period but was statistically significant between ( 0 and 1 month) and between ( 2 weeks and 1 month). $\mathrm{p} \leq 0.001$

\section{For ball retained implant overdenture with soft food chewing:}

For temporalis muscle there was statistically significant decrease in the mean muscle activity through the whole follow up period $\mathrm{p} \leq 0.001$. While for masseter muscle there was statistically significant decrease in the mean muscle activity through the whole follow up period $\mathrm{p} \leq 0.001$.

\section{For ball retained implant overdenture with hard food chewing:}

For temporalis muscle there was non significant decrease in the muscle activity between $(0$ and 2 weeks) on the other hand there was statistically significant decrease in the muscle activity at ( 0 and 1 month) and between ( 2 weeks and 1 month) $\mathrm{p} \leq 0.001$. In case of masseter muscle between ( 0 and 2 weeks) there was statistically significant decrease in the muscle activity $\mathrm{p}<0.01$, also between ( 2 weeks and 1 month) there was non significant difference in the mean muscle activity yet there was statistically significant decrease in the mean muscle activity between ( 0 and 1 month). $\mathrm{p} \leq 0.001$

TABLE (1) Showing the masseter muscle activity $(\mu \mathrm{V})$ with soft and hard food through the follow up periods

\begin{tabular}{|c|l|c|c|c|}
\hline \multicolumn{2}{|c|}{} & At insertion & Two weeks & One month \\
\hline \multirow{3}{*}{ Soft } & Telescopic -retained implant overdenture & $259.42 \pm 12.54$ & $220.28 \pm 6.55$ & $169.71 \pm 10.37$ \\
\cline { 2 - 5 } & Ball-retained implant overdenture & $288.71 \pm 9.89$ & $247.42 \pm 10.45$ & $211.71 \pm 10.02$ \\
\hline \multirow{2}{*}{ Hard } & Telescopic -retained implant overdenture & $250.28 \pm 13.46$ & $216.85 \pm 8.45$ & $172.85 \pm 21.83$ \\
\cline { 2 - 6 } & Ball-retained implant overdenture & $286.14 \pm 7.64$ & $248.57 \pm 11.67$ & $240.71 \pm 31.5$ \\
\hline
\end{tabular}

TABLE (2) Showing the temporalis muscle activity $(\mu \mathrm{V})$ with soft and hard food through the follow up periods

\begin{tabular}{|c|l|r|r|r|}
\hline & & \multicolumn{1}{l|}{ At insertion } & \multicolumn{1}{l|}{ Two weeks } & \multicolumn{1}{l|}{ One month } \\
\hline \multirow{3}{*}{ Soft } & Telescopic -retained implant overdenture & $327.14 \pm 13.35$ & $294.42 \pm 7.56$ & $227.14 \pm 36.01$ \\
\cline { 2 - 5 } & Ball-retained implant overdenture & $313.42 \pm 9.57$ & $288.57 \pm 8.81$ & $235.14 \pm 54.81$ \\
\hline \multirow{2}{*}{ Hard } & Telescopic -retained implant overdenture & $317.71 \pm 10.73$ & $281.57 \pm 11.58$ & $261.28 \pm 18.05$ \\
\cline { 2 - 5 } & Ball-retained implant overdenture & $331.28 \pm 14.48$ & $302.28 \pm 10.27$ & $256.57 \pm 33.59$ \\
\hline
\end{tabular}




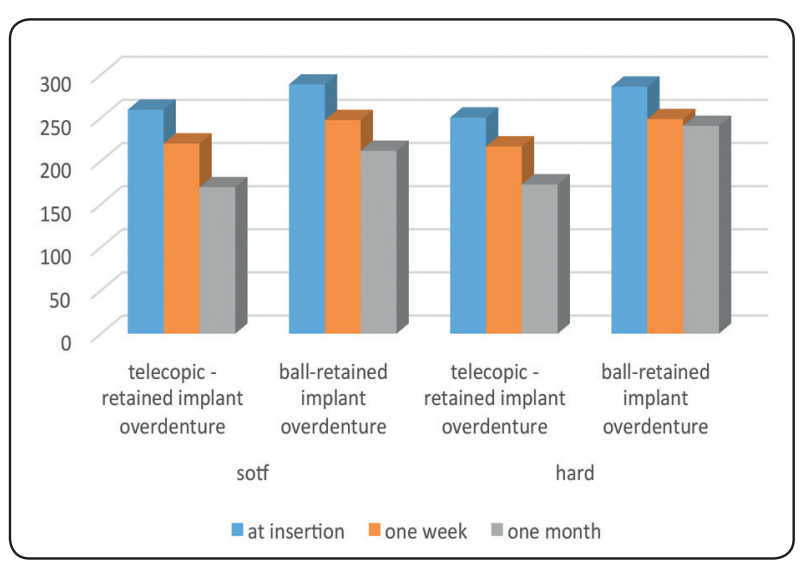

Fig.(3): Changes in masseter muscle activity with soft and hard food in both treatment modalities through the follow up periods

\section{DISCUSSION}

EMG depends on several factors as patients age as well as the psychological factors as fear of pain, fracture, instability of the denture, are considered to have a great influence on muscular activity reduction; it was thought that the efficiency of the muscles of mastication decrease by age however it seemed the same because by aging and loss of teeth the patient exerts more effort to masticate. ${ }^{5}$ Patient's gender; studies revealed that males have more rhythmic and smooth patterns than females ${ }^{6}$ That is why all patients selected were male; the number of functioning teeth, chewing side preference, dental stability and lack of interferences also affects the masticatory muscle activity. ${ }^{7-9}$ Also facial morphology and head posture were found to affect the EMG of masseter and temporalis muscle activity. ${ }^{19,20}$

Therefore temporalis and masseter muscles were chosen for representing the masticatory muscle activity as they are the largest and strongest muscles of the masticatory apparatus as well as being accessible during recording. In fact, neuromuscular control has always been considered as a critical influential factor on the retention and stability of removable prostheses. Different types of chewing tablets consistencies were used to take the records to resemble variation in muscular activity during chewing different types of food.

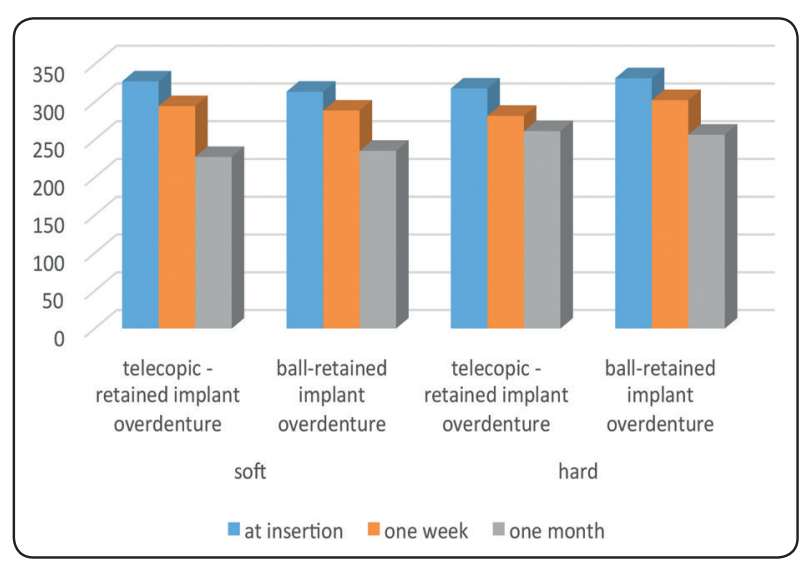

Fig. (4): Changes in temporalis muscle activity with soft and hard food in both treatment modalities through the follow up periods.

The muscle activity was lower in case of telescopic retained implant overdenture compared to the ball implant overdenture through the whole follow up periods and this was attributed to: telescopic attachments are rigid attachments that provides high retention and stability properties of the prosthesis hence less effort is required by the muscles to perform their function. ${ }^{21}$

Also the rigid connection would decrease the possibility of movement of the end part of the overdenture base and so more secured function. On the contrary in the resilient ball attachment the degree and range of movements allowed by the attachment increased the movement of the prosthesis and so this will need more muscle effort exerted..$^{22-24}$

For both groups the muscle activity decreased gradually through the treatment follow up period which is due to the gradual build up of adaptation and confidence in the prosthesis so less effort was required by the muscles to perform their function. ${ }^{25}$

\section{CONCLUSION}

Within the limitations of this study it was concluded that with telescopic retained implant overdentures the muscle activity was lower than ball retained implant over denture. 


\section{CONFLICT OF INTEREST}

No conflict of interest. The study was self -funded by the authors

\section{REFERENCES}

1. Al-Magaleh, W. R.,Abbas, N. A.,Amer, A.A.,Abdelkader, A. A. \& Bahgat, B. Biting force and muscle activity in implant-supported single mandibular overdentures opposing fixed maxillary dentition. Implant Dent. 25, 199-203 (2016).

2. Bakke, M., Holm, B. \& Gotfredsen, K. Masticatory function and patient satisfaction with implant-supported mandibular overdentures: a prospective 5-year study. Int. J. Prosthodont. 15, 575-581 (2002).

3. Koc, D., Dogan, A. \& Bek, B. Bite force and influential factors on bite force measurements: a literature review. Eur. J. Dent. 4, 223-232 (2010).

4. Van Kampen, F. M. C., Van Der Bilt, A., Cune, M. S., Fontijn-Tekamp, F. A. \& Bosman, F. Masticatory function with implant-supported overdentures. J. Dent. Res. 83, 708-711 (2004).

5. Passia, N. et al. Single dental implant retained mandibular complete dentures--influence of the loading protocol: study protocol for a randomized controlled trial. Trials 15 , 186 (2014).

6. Krennmair, G., Weinländer, M., Krainhöfner, M. \& Piehslinger, E. Implant-supported mandibular overdentures retained with ball or telescopic crown attachments: a 3-year prospective study. Int. J. Prosthodont. 19, 164-170 (2005).

7. Do Amaral, B. A. et al. A clinical follow-up study of the periodontal conditions of RPD abutment and non-abutment teeth. J. Oral Rehabil. 37, 545-552 (2010).

8. Tokuhisa, M., Matsushita, Y. \& Koyano, K. In vitro study of a mandibular implant overdenture retained with ball, magnet, or bar attachments: comparison of load transfer and denture stability. Int. J. Prosthodont. 16, 128-134 (2003).

9. Gotfredsen, K. \& Holm, B. Implant-supported mandibular overdentures retained with ball or bar attachments: a randomized prospective 5-year study. Int. J. Prosthodont. $13,125-130$ (2000).

10. Mumcu, E., Bilhan, H. \& Geckili, O. The effect of attachment type and implant number on satisfaction and quality of life of mandibular implant-retained overdenture wearers. Gerodontology 29, (2012).

11. E. Frisch1, P. Ratka-Krüger2, K. M. L. Clinical outcomes of implant-supported and rigidly double crownretained prostheses in edentulous mandibles: An 8-year retrospective study. J. Dent. Implantol. 113-127 (2014).

12. Ohara, Y. et al. Masseter muscle tension and chewing ability in older persons. Geriatr. Gerontol. Int. 13, 372-377 (2013).

13. Brennan, D. S., Spencer, A. J. \& Roberts-Thomson, K. F. Tooth loss, chewing ability and quality of life. Qual. Life Res. 17, 227-235 (2008).

14. Piancino, M. G., Bracco, P., Vallelonga, T., Merlo, A. \& Farina, D. Effect of bolus hardness on the chewing pattern and activation of masticatory muscles in subjects with normal dental occlusion. J. Electromyogr. Kinesiol. 18, 931-937 (2008).

15. De FelÍcio, C. M., Sidequersky, F. V., Tartaglia, G. M. \& Sforza, C. Electromyographic standardized indices in healthy Brazilian young adults and data reproducibility. J. Oral Rehabil. 36, 577-583 (2009).

16. van der Bilt, A. Human oral function: a review. Brazilian J. Oral Sci. 1, 7-18 (2002).

17. Alajbeg, I.Z., Valentic-Peruzovic, M.,Alajbeg, I. \& Cifrek, $M$. The influence of age and dental status on elevator and depressor muscle activity. J. Oral Rehabil. 33, 94-101 (2006).

18. Shaarawy, M. A. \& Aboelross, E. M. The Effect of Varying Implant Position in Immediately Loaded ImplantSupported Mandibular Overdentures. J. Oral Implantol. (2013). doi:10.1563/AAID-JOI-D-12-00098

19. Gomes, S. G. F., Custodio, W., Faot, F., Del Bel Cury, A. A. \& Garcia, R. C. M. R. Masticatory features, EMG activity and muscle effort of subjects with different facial patterns. J. Oral Rehabil. 37, 813-819 (2010).

20. Ceneviz, C. et al. The immediate effect of changing mandibular position on the EMG activity of the masseter, temporalis, sternocleidomastoid, and trapezius muscles. Cranio 24, 237-244 (2006).

21. Heckmann, S. M., Heußinger, S., Linke, J. J., Graef, F. \& Pröschel, P. Improvement and long-term stability of neuromuscular adaptation in implant-supported overdentures. Clin. Oral Implants Res. 20, 1200-1205 (2009). 
22. Elsyad, M. A. \& Khairallah, A. S. Chewing efficiency and maximum bite force with different attachment systems of implant overdentures: a crossover study. Clin. Oral Implants Res. 28, 677-682 (2017).

23. Assunção, W. G., Barão, V. A. R., Delben, J. A., Gomes, É. A. \& Tabata, L. F. A comparison of patient satisfaction between treatment with conventional complete dentures and overdentures in the elderly: A literature review. Gerodontology 27, 154-162 (2010).
24. Hoffmann, O., Beaumont, C., Tatakis, D. N. \& Zafiropoulos, G.-G. Telescopic Crowns as Attachments for Implant Supported Restorations: A Case Series. J. Oral Implantol. 32, 291-299 (2006).

25. Rastogi, A. et al. Electromyographic evaluation of the effect of lined dentures on masticatory muscle activity in edentulous subjects. J. Clin. Diagnostic Res. 9, ZC80ZC83 (2015). 